\title{
Early discharge criteria in patients with acute bacterial skin and skin structure infections in a large tertiary-care teaching hospital in Florence, Italy
}

\author{
Filippo Lagi ${ }^{1}$ (1) - Letizia Ottino ${ }^{1}$ • Elisabetta Mantengoli ${ }^{2}$ Alberto Distefano ${ }^{1} \cdot$ Giampaolo Corti $^{1,2}$ - Alberto Farese $^{2}$. \\ Bassam Dannaoui $^{3}$ - Alessandra Ipponi ${ }^{4} \cdot$ Tiziana Falai $^{4}$ - Gian Maria Rossolini ${ }^{1,5} \cdot$ Alessandro Bartoloni $^{1,2}$. \\ Filippo Bartalesi ${ }^{2}$
}

Received: 14 March 2019 / Accepted: 4 June 2019 / Published online: 20 June 2019

(C) The Author(s) 2019

\begin{abstract}
The study is aimed at retrospectively estimating the percentage of inpatients with severe acute bacterial skin and skin structure infections (ABSSSI) who met the early discharged (ED) criteria adapted from Nathwani et al. (Int J Antimicrob Agents. 2016 Aug;48(2):127-36) and to calculate the number of hospitalization days that could be potentially saved. A retrospective study was conducted in a tertiary care hospital in Florence, Italy. We included all patients admitted for cellulitis and post-surgical infections from 2014 to 2017. Demographic and clinical data were obtained from electronic medical records. We a priori defined the following as a risk factor for non-adherence (RFNA): active or on methadone intravenous drug users, homeless, migrants without health care assistance, and patients who need a caregiver to take prescribed medications. One hundred sixty-two subjects were enrolled. Of them, 94 (58.0\%) were male, and $113(69.7 \%)$ had cellulitis/erysipelas. A microbiological isolate was obtained in 51 patients (31.4\%); Staphylococcus aureus was the most frequent (47\%). Eighty-four (51.8\%) were ED suitable, with $258(49.0 \%)$ patient days potentially saved. Among the 78 not ED suitable patients, the most common reason for prolonged length of stay (LOS) was having at least one RFNA (34.6\%). Fourteen (18.0\%) had one RFNA. Half of the patients admitted in our hospital met the ED criteria with a sparing close to $50 \%$ in terms of hospitalization days. Unstable social and personal factors were the most frequent causes for prolonged LOS. In this selected subset of patients, more recent and easier to administer treatments, including long-acting agents, could be proposed.
\end{abstract}

Keywords ABSSSI · Early discharge $\cdot$ Long-acting $\cdot$ Italy

\author{
Filippo Lagi \\ filippo.lagi@unifi.it \\ Letizia Ottino \\ letizia.ottino@outlook.com \\ Elisabetta Mantengoli \\ mantengolie@aou-careggi.toscana.it \\ Alberto Distefano \\ albertodistefano5@gmail.com \\ Giampaolo Corti \\ giampaolo.corti@unifi.it
Alberto Farese
faresea@aou-careggi.toscana.it
Bassam Dannaoui
dannaouib@aou-careggi.toscana.it
Alessandra Ipponi
ipponia@aou-careggi.toscana.it

Tiziana Falai

falait@aou-careggi.toscana.it

Gian Maria Rossolini

gianmaria.rossolini@unifi.it

Alessandro Bartoloni

alessandro.bartoloni@unifi.it

Filippo Bartalesi

bartalesif@aou-careggi.toscana.it

1 Department of Experimental and Clinical Medicine, University of Florence, Florence, Italy

2 Infectious and Tropical Diseases Unit, Careggi University Hospital, Florence, Italy

3 Health Direction, Careggi University Hospital, Florence, Italy

4 Hospital Pharmacy and Pharmaceutical Policies, Careggi University Hospital, Florence, Italy

5 Clinical Microbiology and Virology Unit, Careggi University Hospital, Florence, Italy 


\section{Background}

In 2013, the United States (US) Food and Drug Administration (FDA) proposed new guidelines and recommendation on developing drugs for the treatment of skin and soft tissues infections, using a new definition of acute bacterial skin and skin structure infection (ABSSSI) [1]. According to FDA, ABSSSI is defined as a bacterial infection of the skin with a lesion size area of at least $75 \mathrm{~cm}^{2}$ and the infection types included are cellulitis, erysipelas, major skin abscesses, and wound infections [1, 2].

ABSSSI is a frequent cause of morbidity and represents a frequent cause of admission to the emergency department [3]. The most common pathogens involved in ABSSSI are typical skin colonizers such as Gram-positive cocci and, in particular, beta-hemolytic streptococci and Staphylococcus aureus [4]. Globally, the high level of resistance to many antibiotics in S. aureus makes the appropriate selection of the antibiotic for physicians highly challenging and a severe threat for patients [5]. Antibiotic-resistant bacteria such as methicillin-resistant S. aureus (MRSA) causing ABSSSI are more challenging to treat due to the lower availability of effective antibiotics and higher mortality rate [5]. The management of ABSSSI often requires intravenous antibiotic therapy and infection-source control through surgical debridement or abscess drainage [6].

The need for intravenous antibiotic therapy, along with an increase of drug-resistant bacteria such as MRSA, both impact on the duration ofhospitalization causing high costs and increasing patient's morbidity and mortality $[7,8]$. Length of hospitalization could be shortened when patients are suitable for early discharge according to standardized clinical criteria for switching to an oral antibiotic [9]. However, in some cases, it could not be feasible because of unstable social and personal factors that would interfere with successful outpatient care [3]. New developed long-acting antibiotics with MRSA activity are recently approved for ABSSSI treatment and could be suitable in this subset of patients.

We report the result of a study conducted in an Italian tertiary care teaching hospital focused on ABSSSI with the purpose to estimate the percentage of inpatients with ABSSSI who could be discharged earlier, based on adapted criteria from Nathwani et al. and calculate the number of inpatient day that could have been saved [9]. We also investigated factors associated with prolonged length of stay.

\section{Methods}

\section{Study setting and patients}

We conducted a retrospective cross-sectional study in Azienda Ospedaliero-Universitaria Careggi (Careggi Hospital), Florence, Italy. Careggi Hospital is a tertiary care teaching hospital with 1297 beds and more than 60,000 hospitalizations per year. From
January 2015 , a multidisciplinary antimicrobial stewardship team operates in Careggi Hospital ensuring the proper use of antimicrobial agents and the adherence to protocols for infection management. However, no defined protocol for ABSSSI was yet in place at the time of the study. The local ethical committee approved the study with the reference number: 11689_oss.

We enrolled all adult patients ( $\geqq 18$ years) hospitalized for cellulitis and surgical site infections selected by diagnosisrelated group (DRG) from January 1, 2014 to December 31, 2017. The DRG codes included in the analysis were: 277 (cellulitis $>17$ years with complications), 278 (cellulitis $>$ 17 years without complications), and 418 (post-surgical and traumatic infections). Demographic, clinical, and laboratory characteristics were extrapolated from electronic medical records on the day of the first evaluation in the emergency department, and on the third day (+ 1 day if no laboratory or clinical data were available in the medical records) of hospitalization. By ABSSSI definition, all patients with one or more of the following conditions were excluded from the analysis: impetigo, burns, minor abscess, bites, necrotizing lesions, diabetic foot, chronic ulcers, septic arthritis, osteomyelitis, pregnancy, neutropenia defined as white blood cells $<1000$ cells/ $\mathrm{mm}^{3}$, and clean contaminated/contaminated wounds surgery. If a patient had more than one hospitalization in the last 12 months, we included only the first episode.

\section{Early discharge criteria and interpretation}

A patient was eligible for early discharge if all the criteria listed in Table 1 were present on day $3(+1)$ of hospitalization. The proportion of hospitalized patients meeting the adapted early discharge criteria from Nathwani et al. was assessed using a dichotomous variable that expressed adherence to the criteria on day 3 ( +1 day). The population was then split into two groups: patients who met early discharge criteria (ED group) and who did not (NED group). The days of hospitalization in excess in ED group criteria were calculated.

When lesion size measurement on day 3 was not reported in medical records, we considered the expression "lesion's clinical improvement" equivalent to the reduction of at least $20 \%$ in lesion size. A stable social and mental condition was interpreted as the possibility of autonomously providing for the withdrawal and correct self-administration of home therapy. We a priori considered the following situations a risk factor for non-adherence: active intravenous drug users (IVDU) or on methadone treatment, homeless, migrants without health care assistance, and patients who need a caregiver due to inability to take prescribed medications on their own (e.g., elderly people with inability for self-care or home transfer, and subjects with psychiatric illness with severe degree of disability). Finally, we considered patients with vomit/diarrhea or any other medical condition that made them unable to swallow (e.g., esophageal or neurological disorders) not eligible for early discharge (Table 1). 
Table 1 Patient eligibility criteria for early discharge in ABSSSI patients (adapted from Nathwani et al.) [9]

I.v. antibiotics for $>24 \mathrm{~h}$

Afebrile (temperature $<38{ }^{\circ} \mathrm{C}$ ) for $>24 \mathrm{~h}$

Clinical lesion improvement of the lesion or stable infection

WBC count of $4 \times 10^{9} / \mathrm{L}$ to $11 \times 10^{9} / \mathrm{L}$

No unexplained tachycardia

Systolic blood pressure $\geq 100 \mathrm{mmHg}$

Patient tolerates oral fluids/diet and can take oral medications with no gastrointestinal absorption problems

No other reason to stay in hospital except for infection management (e.g., surgery scheduled in $48 \mathrm{~h}$; the presence of drainage)

Stable co-morbid illness

No risk factors for non-adherence for the withdrawal and the correct self-administration of home oral therapy:

- Active or former intravenous drug users receiving methadone

- Homeless

- Migrants without health care assistance

- Patients who need a caregiver because not able to take prescribed medications on their own

- Patients with vomit/diarrhea or any other medical condition that makes them unable to swallow

i.v., intravenous; $W B C$, white blood cell; $A B S S S I$, acute bacterial skin and skin structure infections

\section{Statistical methods}

Data were analyzed using the Stata/MP version 14.0 program (StataCorp, College Station, TX). The continuous variables were expressed with median and interquartile range, categorical variables as proportions. The differences between the groups were assayed by Student $t$ or Wilcoxon ranks test where appropriate.

Availability of data and material The datasets used and/or analyzed during the current study are available from the corresponding author on reasonable request.

\section{Results}

We evaluated 229 patients. According to the FDA's ABSSSI definition, 67 patients were excluded (21 immunosuppressed, 13 diabetic foot, 9 missing data, 5 osteomyelitis, 7 prosthetic joint infections, 6 contaminated surgery wound infections, 4 animal bites, 2 pregnancy). The size of the lesion was reported only in nearly $20 \%$ of the medical records. Finally, 162 subjects were included. Demographic and clinical characteristics of the study population are summarized in Table 2 . A microbiological isolate was obtained in 51 subjects (31.4\%), and S. aureus was the most frequent isolate (47.0\%): methicillin-susceptible $S$. aureus (MSSA) in 17 cases $(70.8 \%)$ and MRSA in 7 (29.2\%). Other isolates were: Streptococcus pyogenes $(N=4$; $7.8 \%)$, Streptococcus spp. $(N=6 ; 8.5 \%)$, coagulase-negative
Table 2 Demographic and clinical characteristics of the ABSSSI population (total $N=162$ )

\begin{tabular}{|c|c|}
\hline & $N(\%)$ \\
\hline Male & $94(58.0)$ \\
\hline Female & $68(42.0)$ \\
\hline Age, median (IQR) & $61(43-78)$ \\
\hline \multicolumn{2}{|l|}{ Type of lesion } \\
\hline - Cellulitis/erysipelas & $113(69.7)$ \\
\hline - Wound infection & $28(17.3)$ \\
\hline - Major cutaneous abscess & $21(13.0)$ \\
\hline \multicolumn{2}{|l|}{ Location of ABSSSI } \\
\hline • Lower limb & $85(52.5)$ \\
\hline • Upper limb (no hand) & $16(9.0)(9.8)$ \\
\hline • Hand & $6(3.7)$ \\
\hline • Face & $28(17.3)$ \\
\hline - Groin/sacral area & $10(6.2)$ \\
\hline - Abdomen & $7(4.3)$ \\
\hline - Chest & $10(6.2)$ \\
\hline \multicolumn{2}{|l|}{ Previous episode of ABSSSI } \\
\hline$\cdot$ No & $148(91.0)(91.4)$ \\
\hline - Yes & $14(8.6)$ \\
\hline \multicolumn{2}{|c|}{ Number of hospitalization in the previous 12 months } \\
\hline$\bullet 0$ & $97(59.9)$ \\
\hline$\bullet \geq 1$ & $65(40.1)$ \\
\hline \multicolumn{2}{|l|}{ Charlson comorbidity index } \\
\hline • $0-1$ & $114(70.4)$ \\
\hline$\cdot \geq 2$ & $48(29.6)$ \\
\hline \multicolumn{2}{|l|}{ Previous antibiotic therapy } \\
\hline • No & $100(61.7)$ \\
\hline - Yes & $62(38.3)$ \\
\hline \multicolumn{2}{|l|}{ Number of concomitant medications } \\
\hline • $0-1$ & $59(36.4)$ \\
\hline$\cdot 2-3$ & $31(19.0)(19.2)$ \\
\hline$\cdot>4$ & $72(44.4)$ \\
\hline Median days of hospitalization (IQR) & $7(4-10)$ \\
\hline \multicolumn{2}{|l|}{ Antibiotic therapy at discharge } \\
\hline • No & $25(15.4)$ \\
\hline - Yes & $137(84.6)$ \\
\hline \multicolumn{2}{|l|}{ Empiric anti-MRSA therapy } \\
\hline • No & $102(63.0)$ \\
\hline - Yes & $60(37.0)$ \\
\hline
\end{tabular}

ABSSSI (Acute bacterial skin and skin structure infections); MRSA (methicillin-resistant S.aureus)

staphylococci $(N=4 ; 7.8 \%)$, Enterococcus spp. $(N=4 ; 7.8 \%)$, Escherichia coli $(N=2 ; 3.9 \%)$, Pseudomonas aeruginosa $(N=$ $3 ; 5.9 \%)$, and polymicrobial $(N=4 ; 7.8 \%)$.

Eighty-four (51.8\%) patients were suitable for early discharge (ED group), and 16 of them (19.0\%) had a length of stay $(\mathrm{LOS}) \leq 3$ days. In the ED group, we recorded 527 days 
of hospitalization with 258 days (49.0\%) that could have been potentially saved (median 3 days per patient). Among the 78 patients not suitable $(48.2 \%)$ for the early discharge (NED group), the most frequent reason for prolonged LOS was to have at least one risk factor for non-adherence (27 patients, $34.6 \%$, Table 3). In the NED group, 14 (18.0\%) patients had the sole presence of a risk factor for non-adherence. No difference in age or Charlson comorbidity index was observed between ED and NED groups. Median LOS was 6 (IQR 4-8) days and 9 (IQR 7-12) days in the ED and NED group, respectively $(p<0.001)$.

\section{Discussion}

Applying adapted Nathwani et al. criteria to 162 ABSSSI patients observed in our hospital, 285 patient days could have been saved with a median per patient of 3 days (IQR 1-5). To the best of our knowledge, only the study conducted by Eckmann et al. assessed early switch and early discharge criteria in skin and soft tissue infections in Europe [10]. Although, the Eckmann et al. and our study are scarcely comparable because of a different population enrolled in terms of site of infection (complicated skin and soft tissue infections vs. ABSSSI) and etiology (MRSA vs. all other etiology), our analysis showed a higher percentage of eligible patients for early discharge (34.2\% vs. $51.8 \%)$.

Unfortunately, we cannot determine the level of awareness regarding early discharge criteria among physicians and the retrospective design of our study did not permit to clearly identify the reasons for a missed early discharge in the ED group.

Moreover, the premise of early discharge is not tied only to the clinical improvement and to the patient's ability to take the oral prescribed medications but also depends on the ability to provide supply, preparation, and administration of home therapy autonomously. This aspect, often underestimated in switch studies,

Table 3 Frequency of reasons for not meeting discharge criteria in patients with ABSSSI

$\begin{array}{ll}\text { Presence of at least one risk factor for non-adherence } & 27(34.6) \\ \text { WBC count of } 4 \times 10^{9} / \mathrm{L} \text { to } 10 \times 10^{9} / \mathrm{L} & 24(30.7) \\ \text { No lesion improvement or stable infection } & 19(24.4) \\ \text { Febrile (temperature }>38^{\circ} \mathrm{C} \text { ) for }>24 \mathrm{~h} & 17(21.8) \\ \text { Other reasons to stay in hospital } & 13(16.7) \\ \quad(\text { e.g., surgery scheduled in } 48 \mathrm{~h} \text {; } & \\ \quad \text { the presence of drainage) } & 11(14.1) \\ \text { Unstable co-morbid illness } & 5(6.4) \\ \text { A patient does not tolerate oral } & \\ \quad \text { fluids/diet or/and GI absorption problems } & 3(3.8) \\ \text { I.v. antibiotics for }<24 \mathrm{~h} & 1(1.3) \\ \text { Unexplained tachycardia } & \end{array}$

i.v., intravenous; $W B C$, white blood cell; $A B S S S I$, acute bacterial skin and skin structure infections appeared to be fundamental in the population analyzed, where the most frequent reason for a prolonged LOS in the NED group was the presence of at least one risk factor for non-adherence. IVDU and a psychiatric pathology resulting in a severe degree of disability were the two most common risk factors for nonadherence with $11.5 \%$ and $8.9 \%$, respectively [11]. Patients with IV drug addiction or severe psychiatric conditions (e.g., delirium, dementia, and depressed mood) are known to have an extended hospitalization $[12,13]$. Furthermore, an early discharge in IVDU would offer the advantage of the quick removal of the IV access sometime used for illicit drug injection even in a hospital setting. Interestingly, in the NED group, 14 (18.0\%) patients had no clinical reason to stay in the hospital and were not discharged because of the sole presence of a risk factor for non-adherence.

In this perspective, new long-acting therapeutic possibilities (dalbavancin, oritavancin) recently approved for ABSSSI by the European Medicines Agency (EMA) and the FDA could notably reduce the risk of non-compliance with the antibiotic therapy because of their simple dosage and excellent tolerability [7]. Due to their simple administration and greater tissue penetration, they could alleviate the need for a prolonged hospitalization, especially in those patients that are unreliable with regard to following treatment instructions and meeting follow-up needs or have any medical condition that makes them unable to swallow. Finally, if administrated in the very early phase, it may reduce the need for the placement of indwelling catheters especially in population like IVDU potentially at risk for misuse of the device [7]. Although the initial expense for these new types of long-acting drugs proves to be greater than other solutions, cost-effectiveness studies have shown that the costs are amortized by reducing the need of hospitalization and LOS $[14,15]$. While accurate financial accounting was not possible as part of this study, we envisage substantial savings with this approach. This result has been discussed and endorsed by physicians from infectious diseases, emergency department, internal medicine, surgery, health directorate as well as microbiologist and pharmacists. An internal procedure for ABSSSI management was finally implemented.

This study has several limitations mainly due to its retrospective nature, which could have carried to misclassification of patient exposure and outcome. In fact, a priori criteria could overestimate the true prevalence of risk factors for non-adherence. On the other hand, the proportion of some risk factors could have been underestimated because of a missing report in the medical records (e.g., family and social situation).

\section{Conclusions}

To the best of our knowledge, our research fills a gap in the literature by providing an Italian real-life assessment of ABSSSI, irrespective from the microbiological isolation and taking into the account social and mental situation. Our study showed that about half of the patients admitted met the criteria 
for an early discharge with a sparing of nearly $50 \%$ in terms of hospitalization days. In our opinion, raising awareness among the clinicians on adherence to early discharge criteria may lead to a reduction in the timing of hospitalization. The decline in hospitalization time would improve the patient's quality of life and satisfaction, decrease the risk of contracting an intrahospital infection, and get a reduction in hospital costs, thus going to strengthen and rationalize health spending efficiently. The recent availability of new antibiotics with a better safety profile and easy dosage would ensure appropriate treatment at home after an early discharge also in populations with social problems and not inserted in contexts of support.

Acknowledgments Leonardo Cantasano, Dr. Nicoletta Di Lauria, Dr. Stefano Grifoni, Prof. Giacomo Laffi, Prof. Alberto Moggi Pignone, Dr. Alessandro Morettini, Dr. Fabrizio Niccolini, Dr. Carlo Nozzoli, Prof. Loredana Poggesi, Prof. Andrea Valeri, Dr. Riccardo Viligiardi, Dr. Lorenzo Roberto Suardi.

We would like to thank OPT srl and in particular Dr. Camilla Taglietti for methodological support and technical advice.

Authors' contributions FL and FB contributed to the study design and implementation of the research. FL, FB, EM, AF, and GC participated in analysis and interpretation of data. $\mathrm{FL}, \mathrm{AB}, \mathrm{FB}$ participated in the drafting of the manuscript. $\mathrm{LO}, \mathrm{AD}$, and $\mathrm{BD}$ participated in the acquisition of data. $\mathrm{AI}, \mathrm{TF}$, and GMR contributed to the critical revision. All authors read and approved the final manuscript.

\section{Compliance with ethical standards}

Conflict of interest Filippo Bartalesi, Giampaolo Corti, Bassam Dannaoui, Alberto Distefano, Tiziana Falai, Alessandra Ipponi, Filippo Lagi, Elisabetta Mantengoli, Letizia Ottino: the authors declare that they have no competing interests. Alessandro Bartoloni has received fees for presentation at local congress or expert meeting from Pfizer and MSD Italia. Alberto Farese has received fees for presentation at local congress or expert meeting from Becton-Dickinson and MSD Italia. Gian Maria Rossolini received grant and personal fees from Basilea, Merck, Nordic Pharma, Shionogi, Menarini; received personal fees from Pfizer and Qpex and non-financial support from Menarini; received fees for his hospital department from Venatorx.

Ethics approval and consent to participate The local ethical committee (Comitato Etico Area Vasta Centro) approved the study with the reference number: 11689 oss.

\section{Consent for publication Not applicable.}

\begin{abstract}
Abbreviations ABSSSI, acute bacterial skin and skin-structure infection; DRG, diagnosis-related group; $\mathrm{ED}$, early discharge; ED group, patients who met early discharge criteria; FDA, Food and Drug Administration; IVDU, intravenous drug users; MRSA, methicillin-resistant $S$. aureus; MSSA, methicillin-susceptible $S$. aureus; NED group, patients who did not meet early discharge criteria
\end{abstract}

Open Access This article is distributed under the terms of the Creative Commons Attribution 4.0 International License (http:// creativecommons.org/licenses/by/4.0/), which permits unrestricted use, distribution, and reproduction in any medium, provided you give appropriate credit to the original author(s) and the source, provide a link to the Creative Commons license, and indicate if changes were made.

\section{References}

1. Food and Drug Administration. Guidance for industry acute bacterial skin and skin structure infections : developing drugs for treatment. 2013;

2. Falcone M, Concia E, Giusti M, Mazzone A (2016) Acute bacterial skin and skin structure infections in internal medicine wards: old and new drugs. Intern Emerg Med. 11(5):637-48

3. Pollack CV, Amin A, Ford WT, Finley R, Kaye KS, Nguyen HH et al (2015) Acute bacterial skin and skin structure infections (ABSSSI): practice guidelines for management and care transitions in the emergency department and hospital. J Emerg Med 48(4):50819

4. Esposito S, Bassetti M, Concia E, De Simone G, De Rosa FG, Grossi P et al (2017) Diagnosis and management of skin and softtissue infections ( SSTI ). A literature review and consensus statement : an update. J Chemother 29(4):197-214

5. Pulido-Cejudo A, Guzmán-Gutierrez M, Jalife-Montaño A, OrtizCovarrubias A, Martínez-Ordaz JL, Noyola-Villalobos HF et al (2017) Management of acute bacterial skin and skin structure infections with a focus on patients at high risk of treatment failure. Ther Adv Infect Dis 4(5):143-161

6. Stevens DL, Bisno AL, Chambers HF, Dellinger EP, Goldstein EJC, Gorbach SL, et al. Practice guidelines for the diagnosis and management of skin and soft tissue infections : 2014 update by the Infectious Diseases Society of America. 2014

7. Russo A, Concia E, Cristini F, De RFG, Esposito S, Menichetti F et al (2016) Current and future trends in antibiotic therapy of acute bacterial skin and skin-structure infections. Clin Microbiol Infect 22:S27-S36

8. Bassetti M, Eckmann C, Peghin M, Carnelutti A, Righi E (2018) When to switch to an oral treatment and/or to discharge a patient with skin and soft tissue infections. Curr Opin Infect Dis 31:163169

9. Nathwani D, Dryden M, Garau J, Hampshire R, Hospital C, Road R et al (2016) Early clinical assessment of response to treatment of skin and soft tissue infections. Int J Antimicrob Agents. 48(2):12736

10. Eckmann C, Lawson W, Nathwani D, Solem CT, Stephens JM, Macahilig C et al (2014) Antibiotic treatment patterns across Europe in patients with complicated skin and soft-tissue infections due to meticillin-resistant Staphylococcus aureus: a plea for implementation of early switch and early discharge criteria. Int J Antimicrob Agents 44:56-64

11. Cyriac J, James E (2014) Switch over from intravenous to oral therapy: a concise overview. J Pharmacol Pharmacother 5:83

12. Chotai N, Gallagher K, Kieffer WKM, Rogers BA, Ricketts DM (2016) A comparative analysis of the patient characteristics and cost of managing intravenous drug users (IVDU) with soft tissue abscesses with non-IVDU. Surgeon 14:13-17

13. Saravay SM, Lavin M (1994) Psychiatric comorbidity and length of stay in the general Hospital: a critical review of outcome studies. Psychosomatics 35:233-252

14. Nair T, Fitzgerald J, Ly B, Wallace MR (2018) Dalbavancin as a cost effective antibiotic. Infect Dis (Auckl) 50:75-76

15. Agarwal R, Bartsch SM, Kelly BJ, Prewitt M, Liu Y, Chen Y et al (2018) Newer glycopeptide antibiotics for treatment of complicated skin and soft tissue infections: systematic review, network metaanalysis and cost analysis. Clin Microbiol Infect 24:361-368

Publisher's note Springer Nature remains neutral with regard to jurisdictional claims in published maps and institutional affiliations. 